\title{
Treatment considerations for OAB: Case studies
}

\author{
Sidney B. Radomski, MD, FRCSC;; Jane Schulz, MD, FRCSC ${ }^{+}$
}

*Professor of Surgery (Urology), University of Toronto, and Director of the Urodynamics Laboratory, Toronto Western Hospital-University Health Network, Toronto, ON; ‘'Associate Professor of Obstetrics and Gynecology, University of Alberta, and Urogynecologist, Lois Hole Hospital for Women, Royal Alexandra Hospital, Edmonton, AB

Cite as: Can Urol Assoc J 2013;7(9-10):S177-8. http://dx.doi.org/10.5489/cuaj.1616 Published online October 9, 2013.

\section{Abstract}

As part of the program at the $3^{\text {rd }}$ Annual Canadian Urology Forum (2013), participants engaged in interactive discussions of difficult cases throughout the event. The following is a summary of discussions pertaining to two cases illustrating treatment considerations in overactive bladder.

\section{Case 1}

\section{A 77-year-old man with Parkinson's disease, urgency and urgency incontinence}

Mr. AR is a 77-year-old man with an eight-year history of Parkinson's disease. He presents complaining of nocturia (3-4 times nightly), frequency (approximately every two hours), urgency and urgency incontinence. He mentions that he also experiences periodic, mild constipation.

Nine years ago, he underwent transurethral resection of the prostate (TURP) following the development of benign prostatic hypertrophy and associated lower urinary tract symptoms. A trial of alpha-blocker (tamsulosin) therapy was unsuccessful due to the development of dizziness.

At the time of presentation, his medications included a 5-alphareductase inhibitor (dutasteride) and his anti-Parkinsonian medications (levodopa and the dopamine agonist pramipexole). He indicated that he limits fluid intake and avoids caffeine.

Cystoscopy and uroflow were performed, showing some residual prostate and mild obstruction. There was a mild post-void residual (PVR), a uroflow of $8 \mathrm{~mL} / \mathrm{sec}$, and $211 \mathrm{~mL}$ of voided volume. At that time, he was prescribed long-acting tolterodine $4 \mathrm{mg}$ daily and told that if this did not improve his symptoms, further investigations would be conducted (video urodynamics [UDS]).
A few months later, his neurologist initiated the cholinesterase inhibitor donepezil, $10 \mathrm{mg}$ daily.

Seven months after the last visit to the urologist, the patient was admitted to hospital with a decreased level of consciousness and behaviour problems. The attending neurologist discontinued the tolterodine and he was discharged with no overactive bladder $(\mathrm{OAB})$ medication. Subsequent to this, the patient noted an increase in incontinence episodes and he resumed tolterodine therapy on his own while waiting for his next appointment with the urologist.

At the next urology appointment, video UDS demonstrated a PVR of $25 \mathrm{~mL}$ at the start. With filling, normal sensation and compliance were reported, but there was difficulty filling due to leakage and instability $\left(60 \mathrm{~cm} \mathrm{H}_{2} \mathrm{O}\right)$. The urologist was unable to get an adequate flow rate and pressure, and it was difficult to assess the bladder neck. The PVR at the end of the study was again only $25 \mathrm{~mL}$.

\section{Discussion}

The participants were asked to voice their opinions on the way this case was handled and how they might proceed at this stage of the patient's management. One of the key elements discussed was the role of the antimuscarinic in the patient's symptoms of dementia. The participants agreed that in this scenario, using an antimuscarinic with a lower incidence of central nervous system effects (e.g., darifenacin) would be advisable. However, it was mentioned that the effects of these agents would be attenuated if co-administered with a cholinesterase inhibitor.

Botulinum toxin or mirabegron were alternatively suggested as potential options to diminish this patient's urinary symptoms without the potential hazards of anticholinergic activity. Nonpharmacologic counselling and assisted living were also proposed as possibly beneficial approaches to this patient's urinary tract symptoms.

There was some disagreement with respect to the attempt to use an alpha-blocker after TURP. Some participants argued that it can provide some benefit, depending on the individual patient's symptom profile, while others disagreed with the practice, citing the lack of alpha receptors to target in that setting. 


\begin{tabular}{cccc}
\hline Table 1. Voiding diary for patient \#2, a 70-year-old woman with worsening urgency and urge incontinence \\
\hline Time & Intake & Void & Leak \\
\hline 0700 & 2 cups coffee & $300 \mathrm{~mL}$ & On way to bathroom (L) \\
$0800-N o o n$ & 2 glasses iced tea & 4 voids $50-100 \mathrm{~mL}$ & 2 leaks on way to bathroom \\
Noon-1600 & 3 rum and cokes & 3 voids 100-150 mL & 1 leak on way to bathroom \\
$1600-2100$ & 1 glass water & 4 voids $100 \mathrm{~mL}$ & 1 leak on way to bathroom \\
After bedtime & 3 small voids & 1 leak on way to bathroom \\
\hline
\end{tabular}

\section{Case 2}

\section{A 70-year-old woman with worsening urgency and urge incontinence}

The patient is a retired 70-year-old woman with a two-year history of progressively worsening urgency. She has hypertension (treated with the calcium channel blocker amlodipine $10 \mathrm{mg}$ daily), and her history includes a hysterectomy (for menorrhagia) in her early 40s.

For the past six months, she has begun to have episodes of urge incontinence. She consistently experiences urge and leakage during sexual intercourse. She has daytime frequency (average of 10 daytime voids) and nocturia (average of three voids nightly). There are no complaints of prolapse or bowel dysfunction.

In the physical examination, she does not exhibit stress urinary incontinence (SUI) on cough test with a full bladder, there is slight atrophy, no prolapse, and no pelvic masses. Urinalysis is negative.

Over the course of her treatment, she tried strengthening pelvic floor muscles with Kegel exercises, but derived no benefit from this intervention. Her initial pharmacotherapy was oxybutynin, but she discontinued this medication due to poor tolerability. She subsequently experienced a slight improvement with extendedrelease tolterodine, but at this visit, she indicates that she has lost her response to this medication.

For this visit, she was asked to keep a voiding diary. One day of this diary is shown in Table 1.

\section{Discussion}

The participants were asked to comment on how they might proceed at this stage of the patient's management. Several participants discussed the importance of patient education and setting realistic treatment expectations. The suggestions for therapy included first conducting additional testing to confirm the diagnosis is correct; adding adjunctive topical vaginal estrogen to address the urgency; switching to a different antimuscarinic; and switching to mirabegron. Posterior tibial nerve stimulation was presented as another possible option. Botulinum toxin was also discussed as a possibility, as long as the patient was considered healthy enough, was informed of the risk of urinary retention, and was considered capable of self-catheterization.

Competing interests: This article is part of a CUAJ supplement sponsored by Astellas Pharma Canada, Inc. Dr. Radomski is an ongoing paid consultant with Allergan, Astellas, Eli Lilly, Merus Labs, Pfizer, and Watson. He has also received speaker fees, educational grants, and/or travel assistance from Allergan, Astellas, Eli Lilly, and Pfizer within the last two years. Dr. Schulz is an ongoing paid consultant with Astellas, Gynecar, and Pfizer. She has received speaker fees, educational grants, and/or travel assistance from Astellas, Bard, Gynecare, Pfizer, and Triton.

Correspondence: Dr. Sidney B. Radomski, Toronto Western Hospital, Main Pavilion, 8th Floor Rm. 304, 399 Bathurst St., Toronto, ON M5T 2S8; sidney.radomski@uhn.ca 